\title{
Improving Efficiency in IBM Asset Management Software System "Maximo": A Case Study of Dubai Airports and Abu Dhabi National Energy Company
}

\author{
Mounir M. El Khatibํㅜ, Gouher Ahmed² \\ ${ }^{1}$ Hamdan Bin Mohammed Smart University, Dubai, UAE \\ ${ }^{2}$ Skyline University College, Sharjah, UAE \\ Email:drmunir.k@gmail.com, gouher@usa.net
}

How to cite this paper: El Khatib, M.M. and Ahmed, G. (2018) Improving Efficiency in IBM Asset Management Software System "Maximo": A Case Study of Dubai Airports and Abu Dhabi National Energy Company. Theoretical Economics Letters, 8, 1816-1829.

https://doi.org/10.4236/tel.2018.810119

Received: March 12, 2018

Accepted: June 18, 2018

Published: June 21, 2018

Copyright $\odot 2018$ by authors and Scientific Research Publishing Inc. This work is licensed under the Creative Commons Attribution International License (CC BY 4.0).

http://creativecommons.org/licenses/by/4.0/

\begin{abstract}
The study is a study of the asset management of the IBM Maximo with reference to Dubai Airports and Abu Dhabi National Energy Company in the United Arab Emirates, with the top companies in the region and the world at large calling for maximum efficiency and productivity in their asset management. The method of hypothesis testing is followed with the help of other studies' insights on the problem, interviews and secondary data. The reputed IBM's Maximo asset management software is found wanting in asset management in respect of planning and balancing between maintenance and operations. As a result, the Maximo couldn't maximize efficiency of its reputed UAE clients. As a way out, dynamic and flexible solutions of problem solving are suggested, and also one spare and one procurement to save time, space and money. Efficiency enhancement is a must for a well competitive UAE economy.
\end{abstract}

\section{Keywords}

IBM’s Maximo, Asset Management Software System, UAE

\section{Introduction}

The United Arab Emirates, a union of seven Emirates which was formed in 1971, has made rapid economic strides in a relatively short span of time due to the exceptional vision and leadership of its founding fathers H.H. Sheikh Zayed bin Sultan Al Nahyan of Abu Dhabi and H.H. Sheikh Rashid bin Saeed Al Maktoum of Dubai and subsequent leadership that is continuing their leadership 
legacy of putting the UAE at the forefront of economic development as a model Arab State, is well known to be intent on excellence and efficiency in every line of industrial and business activity [1] [2] [3]. In the Emirates, Abu Dhabi and Dubai are the first and second important emirates, respectively, are known for their "public" mega projects in which this study's sample projects of Dubai Airports and Abu Dhabi National Energy Company are important in Aviation and energy, respectively, wherein, efficiency and productivity much to their performance.

The Engineering Service Units at Dubai Airports and Abu Dhabi National Energy Company, which includes seven power and water plants, are known for their business excellence, have further taken-up to using "Maximo", The IBM's asset management software to manage their resources in a more efficient way. The Maximo system is a type of information system (IS) that is based on developing communication, sharing documents, sharing expertise and knowledge, service requests, and complaints, resulting in a way to help in providing a link between cross functional departments and teams through all levels of the corporation, through work orders and work notifications, and tracking and approval patterns which are visible to managers, engineers, supervisors, and technicians enabling a single click of a button from the planner to transfer sufficient manpower and materials directly from suppliers to execute the task.

The Maximo centralizes all of the company's engineering unit functions in an organized pattern to preview the number of assets, manpower, materials, job plans, planning and quality checks, material requisition, compliance of each department tasks, and on analytical report all under a single roof. Maximo enabled the departments to retrofit their available resources to achieve a higher performance through standardizing of task and service levels with a unique planning sequence and tracking features.

The contribution of this study is the investigation into the efficiency aspects of the two major undertakings of the United Arab Emirates where in the IBM Maximo is in application, and suggesting the means of optimizing the efficiency/productivity levels in the two firms through better resource management. It is also brought out that the Maximo is not the ultimate solution to better resource management and higher efficiency. The study also extends to a better Maximo management.

As any study, this study too has the limitations of time and data, which are ever changing being in a state of flux in the dynamic world of technology and business. It is, in other words, a point of time study, not an over a time study. The way for higher levels of productivity in both the firms may not be in Maximo, but a better management model leadership, a motivated labor force, or even labor participation in management. There can never be all-time solutions to the efficiency problem.

The study is organized as follows. First, there is the statement of the problem which is finding out the improvement in the efficiency levels in the two "firms" 
after the introduction of the IBM's "Maximo" is resource management system, which is taken to be efficiency enhancing. Next is a review of literature on industrial and business efficiency and resource managements with any gaps therein. In this section, two hypotheses, $\mathrm{H} 1 \& \mathrm{H} 2$ are formulated and studies examined on them.

\section{Statement of the Problem}

Prior to introducing an IT based software, manual patterns took place and procurement were subjective to each department as well as planning of maintenance regimes in absence of proper record of manpower and craft utilization. Emerging out of a sheer requirement to control and manage resources much more efficiently, centralized systems were introduced to lead and manage business processes through an integrated IBM software called Maximo. The system is newly implemented and after cycles of operations, gaps are noticed and are to be filled with improvement measures, as the system limitations provide better efficiency solutions for retrofits. Hence the problems that are addressed in the study are as follows:

1) Fixed Scheduled maintenance regimes for assets that are aging in time and encountering defects.

2) Common spare parts grouping adds more data points and processes, as they are dealt with as different parts due to ownership.

\section{Literature Review}

Creating a strategy on how to improve the efficiency of Maximo is critical for the Dubai Airports and the Abu Dhabi National Energy Company. Creating a comprehensive grouping of different items from various departments with minimum quantities, financial standing, budget, procurement teams, and the buffer store locations has been studied by several studies as a means of improving the Maximo information system. This section is a literature review of various studies on the hypothesis stated. The scope will analyze how the idea will lead to the efficient operations of the Dubai Airport and the Abu Dhabi National Energy Company.

H1. Additives of health trends such as sensors \& gadgets, and aging factors integrated into the Maximo registered assets will support and enhance the efficiency \& reliability of the assets through centralized planning team.

Centralized departments are a part of the Maximo package. It has been looked after in the two organizations which are Dubai Airports and Abu Dhabi National Energy Company, including its seven, power and water plants across the country.

Many scheduling systems starting from an outlook calendar to a sophisticated IBM software's are pure fixed frequency induced and depend solely on manual timely inputs only. Since meetings can be pushed back and forth with no implications on operations, while maintenance of a rotating machine are of a different 
nature and will have implications on operations, if failure symptoms are present prior to the due date or if delayed after due date. Intelligent Maintenance Systems means are introduced to collect data to predict future failures; through analyzing the behavior of the machine through gadgets/sensors that shifts from fixed maintenance regimes to flexible "Smart" maintenance schedules [4]. Maximo would benefit from this integration, as the current system is not capable of understanding such requirements and adapting modern flexible maintenance regimes is the next step towards enhanced efficiency and reliability though the investment in programing and customizing Maximo to reach towards this goal, which is not yet explored by IBM.

Rotating machinery is part of our daily routine, starting with the vehicle we drive to work has a maintenance schedule. For example, after 10,000 Km or year, whichever comes first in this case is set towards a fixed scheduled maintenance. But all drivers are not the same, some have short trips, some drive in a dusty environment, etc., which affects the condition of the engine oil; therefore users can switch to flexible maintenance that calculates the remaining oil life based on the engine operation condition which then gives you an indication when an oil change is due, and independent on mileage cut it could be less or more than $10,000 \mathrm{KM}$ through the usage of alarming gadgets, and such an approach is known as the flexible maintenance, which not only does serve a better machine life cycle, but also saves the machine from operating till failure mode prior to reaching the scheduled fixed service. By introducing vibration analysis and temperature sensors on the machines, and reporting directly to its registered asset in Maximo, it would serve the purpose and acts towards reaching the asset to its flexible maintenance regime through creating alarms that trigger an early maintenance if required, especially for aging assets and those of which encounter defects through time.

Shifting to another field of concern, ones with heart diseases may encounter serious issues, but unknowingly, due to absence of pain or any other symptom, it is only observed once a diagnosis is performed which acts as a fixed scheduled visit, once per year dependent on individuals; therefore the more the problem prolongs, the more the severity increases. Introducing wearable sensor gadgets that are capable of measuring one's heart rate, breathing pattern, and body temperature will therefore induce an alarming notice of abnormalities that individuals should worry about and seek a doctor visit. Again, a flexible scheduled visit outperforms a scheduled one as it captures alarming points and recommends check-ups prior to due dates, which is a critical issue to human beings' wellbeing [5]. Relating to the subject, Figure 1 \& Figure 2 of the medical and engineering fields, respectively indicate a problem at a point of time/frequency, which trend integration into Maximo would trigger an alarm and a notification to observe and test that particular asset, since it is having problems prior to reaching the maintenance due date, to provide a better life cycle of the asset and save it from failing and reaching to its breakdown point. 
In short, adapting flexible systems means importing alarming gadgets into the system and integrating them to Maximo to free the software from the fixed scheduled maintenance, flexible systems reduce manufacturing expenses [6].

From a view and review of literature on the budding problem it is obvious that there are no country-wise and industry-wise empirical or investigative studies on the problem, especially with reference to the efficiency maximizing Maximo. This study to certain extent fills the literature gap on the issue, it being country, industry and Maximo specific.

The need for carrying out more Maximo studies in the sense of ways and means of better resource management and efficiency even in respect of the dynamic State of the UAE needs no argumentation. This study is a modest starting point in the direction. In aviation and energy, the state stands shall and also in respect of many fields and firms, which can be investigated in respect of the Maximo angle by the future researchers of the problem in the light of enhanced interest in business excellence and greater efficiency levels as a forward business state.

Ideally, centralized planning departments support the main cause of improving the efficiency of systems such as the case in health care facilities, through linking the needs of various services to patients [7] [8]. Similar is the case in the dental field, wherein centralized systems have improved the efficiency through enhancing the communication means according to [9] $85 \%$ of the dental facilities are using centralized systems.

According to Doyle et al., (2014), "Enterprise Asset Management addresses the entire lifecycle management of physical assets of an organization in order to maximize value". Enterprise Asset Management helps to improve utilization, performance, reduce capital costs, reduce operating costs, extending assets life, and improve returns on assets. The IBM-Maximo Asset Management System enables the organizations to schedule work orders and tasks properly and in an organized way that enables them to have a proper prioritization of the work and also contribute to the decrease in the as set's down time for maintenance [10].

Thus, $\mathrm{H} 1$ is vindicated by the afore mentioned studies and also by the interviewed opinions, which are opinions from the field, as well as Figure $1 \&$ Figure 2 variables depictions, as industrial and business conditions are fluctuating over time and space.

H2. Grouping common items from different departments, which have different maximum/minimum quantities, budget, financial standing, procurement teams, and buffer store locations, will improve end user usability.

Air Force has invested substantially in spare parts that are readily made available depending on the maximum/minimum quantity required, through forecasting future requirements from historical trends of NSN (National Stock Numbers) that help the Air Force to allocate a separate budget account for its utilization [11]. Many organizations depend on frequency based condition such as fast moving items, slow moving items, and non-moving items. Yet the 
HR During Interstellar

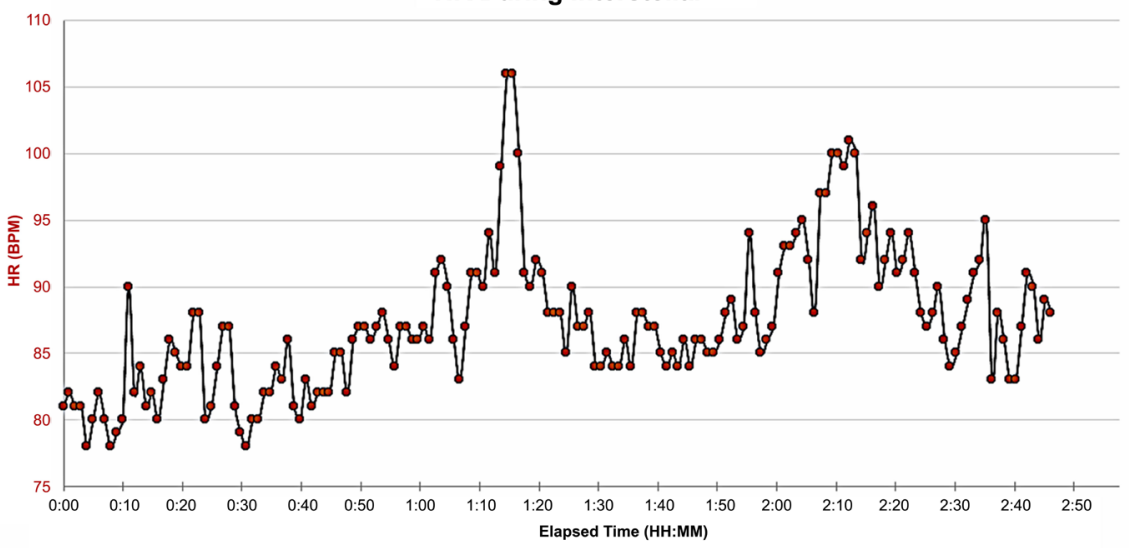

Figure 1. Heart Rate During Interstellar. Source: Figure 1, captured from the medical field of the Engineering Service Units of Abu Dhabi National Energy Company from Integrated IBM Software Maximo in September, 2015. Heart rate beats per minute shows a peak and alarming point at 1.15 minutes.

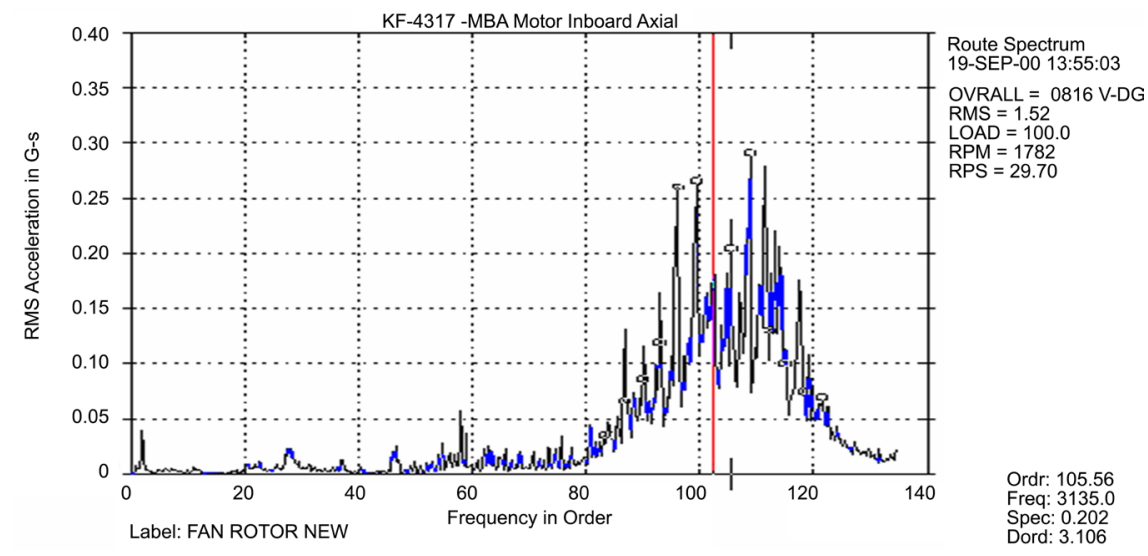

Figure 2. Vibration in the Frequency Order. Source: Figure 2 is captured from engineering field of the Engineering Service Units of Abu Dhabi National Energy Company from integrated IBM Software "Maximo" in September 2015. An increase in the vibration in the frequency order at $115 \mathrm{HZ}$, an alarming point which requires investigation at that particular frequency.

complication arises when grouping those items to different departments with different budget allocations. A similar issue is encountered in Maximo, where maximum/minimum quantity bases are looked after, yet it differs in aspect as it depends on different budgets for departments rather than a single budget.

In the medical field, different surgery departments use similar tools and equipment, which vary in quantity according to the number of patients admitted for surgery in each ward, following which the budget is distributed accordingly with a centralized procurement team. The issue rises where fast moving items needed by departments on an emergency basis shift the dynamic balance of maximum/minimum quantity per department, and a loan based issuance increases the imbalance and reflects on the audit per department at the end of the year [12]. Similarly, in the automobile business where a common spare part of a 
different vehicle is used, management may declare the utilizations of a software to aid in the procurement of such common parts, but depending on the number of vehicles sold to customers, the first model outweighs the second model where the second model, consumes more of the common spare part. However, the software does not accept exceeding the number of spare parts ordered for the second model, due to the number of vehicles sold to customer limitations. Digging deeper into the problem, the inventory software treats the common spare part as two different items for both models occupying more space [13].

Figure 3 describes the same situation in Maximo where it identifies the same spare with a unique identification number as it belongs to a different department, experiencing the same problem of inventory keeping in a different location.

On an organizational level of a telecommunication department of the two organizations with a SLA (Service Level Agreement) a similar issue was dealt with, as a loan based issuance which abides the organizations to compliance of budget transfers by the end of each year and affects the expenditure dramatically in the last months of the year to avoid penalties, adding the process complications which is unfortunately not flexible [14]. Maximo is not directly related to this situation, but since inventory is part of Maximo, central store managers tend to release and override the system and issue the same from different department belongings in order to cope with the emergency requirement and avoid delays which may impact stakeholders and external customers.

Harrington (2015), stated that "IBM Enterprise Asset Management provides predictive inventory optimization to help organizations make inventory savings and protect against out-of-stock conditions for mission-critical parts" [15]. IBM Maximo Asset Management System can be used as a tool that can identify and understand the usage pattern of the assets in order to project and predict the future usage of the assets [16] [17].

Also, seconded by the interviewee when asked about the proposed solution, it was a mixture of managerial and software re-programming, and a door kept open for continuous improvement which attains the corporate requirements and

\begin{tabular}{|c|c|c|c|c|c|}
\hline \multicolumn{2}{|c|}{ 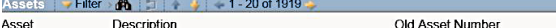 } & Old Asset Number & Functional Location & Parent & Primary Locatic \\
\hline & $a[$ & & & & \\
\hline DA142167 & STARTER, ELEC TRIC MOTOR; PANEL & CUC4-CHWP-STP-1-4 & CUC4-CHWP-STP-1-4 & DA141966 & 08-UC4-0005 \\
\hline DA142168 & STARTER, ELECTRIC MOTOR; PANEL & CUC4-CHWP-STP-2-1 & CUC4-CHWP-STP-2-1 & DA141967 & 08-UC4-0005 \\
\hline DA142169 & STARTER, ELECTRIC MOTOR; PANEL & CUC4-CHWP-STP-2-2 & CUC4-CHWP-STP-2-2 & DA141968 & $08-U C 4-0005$ \\
\hline DA142170 & STARTER, ELECTRIC MOTOR; PANEL & CUC4-CHWP-STP-2-3 & CUC4-CHWP-STP-2-3 & DA141969 & $08-U C 4-0005$ \\
\hline DA142171 & STARTER, ELECTRIC MOTOR; PANEL & CUC4-MCT-STP-1-1 & CUC4-CT-STP-1-1 & DA142092 & 08-UC4-5006 \\
\hline DA142172 & STARTER, ELECTRIC MOTOR; PANEL & CUC4-MCT-STP-1-2 & CUC4-CT-STP-1-2 & DA142093 & 08-UC4-5006 \\
\hline$\overline{\mathrm{DA} 142173}$ & STARTER, ELECTRIC MOTOR; PANEL & CUC4-MCT-STP-1-3 & CUC4-CT-STP-1-3 & DA142094 & $08-U C 4-5006$ \\
\hline DA142174 & STARTER, ELECTRIC MOTOR; PANEL & CUC4-MCT-STP-1-4 & CUCA-CT-STP-1-4 & DA142095 & $08-U C 4-5006$ \\
\hline DA142175 & STARTER, ELECTRIC MOTOR; PANEL & CUC4MCT-STP-2-1 & CUCA-CT-STP-2-1 & DA142096 & $08-U C 4-5006$ \\
\hline DA141488 & PUMP; CNW; 1470 RPM; 381.6 M3:H; 16 BAR & GSEM-CNW-PMP-D3 & GSEM-CNW-PMP-D3 & & 05-USB-1310 \\
\hline DA141489 & $\begin{array}{l}\text { MOTOR, ELECTRIC; CNWF; } 37 \mathrm{KW} ; 380-415 \\
\text { VAC; 65-71 A; } 50 \mathrm{HZ} ; 1470 \mathrm{RPM} ; \mathrm{S1} \text { '; } \\
\text { IP55; F }\end{array}$ & GSEM-CNW-PMP-MTR-01 & GSEM CNW-PMP-MTR-01 & DA141486 & 05-USB-1310 \\
\hline DA141490 & $\begin{array}{l}\text { MOTOR, ELECTRIC; CNWP; } 37 \mathrm{KW} ; 380-415 \\
\text { VAC; 65-71 A; 50 HZ; } 1470 \text { RPM; S1; } \\
\text { IP55; F }\end{array}$ & GSEM-CNW-PMP-MTR-02 & GSEM-CNW-PMP-MTR-02 & DA141487 & 05-USB-1310 \\
\hline$\underline{\text { DA141491 }}$ & $\begin{array}{l}\text { MOTOR, EIECTRIC; CNWF; } 37 \mathrm{KW} ; 380-415 \\
\text { VAC, } 65571 \mathrm{~A} ; 50 \mathrm{HZ} ; 1470 \mathrm{RPM} ; \mathrm{Si} \\
\text { PP55; F }\end{array}$ & GSEM-CNW-PMP-MTR-03 & GSEM-CNW-PMP-MTR-03 & DA141488 & 05-USB-1310 \\
\hline
\end{tabular}

Figure 3. Unique Asset Number with Identical Description. Source: Figure 3 is captured from the engineering field of the Engineering Service Units of Dubai Airports from Integrated IBM software "Maximo" in August 2015. 
special needs justifies the satisfaction of the senior management and the vendor provider IBM. The hypothesis is made feasible and possible due to the vendor capability of introducing new features to the software.

\section{Methodology}

The methodology of the study is to make the Maximo dynamic and to get the better asset management results. Also, hypotheses which are $\mathrm{H} 1$ and $\mathrm{H} 2$, and testing them through the subject literature and primary investigation through executive interview samples of both the firms, Dubai Airport and Abu Dhabi Energy Company.

\subsection{Sources of Data}

Personal communication through Interviews were considered as a qualitative method of data collection where the interviewer asks direct questions to the interviewees regarding specific issues for the sake of collecting information about the issue. It is considered as a primary data collection method.

Engineer Riaz Mubarak from SCADA department \& Engineer Siddique Nizzam from Power Generation department were interviewed about setting the frequency in Maximo. Also, Mr. Adil from procurement, Mr. Hiren from Finance, and Mr. Srinivas from Business support were interviewed on how Maximo can integrate common spare parts for different departments under one grouping (See Appendix).

The interviewees were chosen for their subject expertise. They were, representatives of sample companies in their field. Their voice represents the voice of their companies and they had no personal views to air. They were not given a free field, but asked pointed questions to which they could only give pointed responses based on facts. Hence, the interview data can be treated as quite authentic, with no scope for personal biases.

Hence, the quality and authenticity of data is well taken care of as neither the research/ author interviewer nor the interviewee had no personal interview in the problem, except ascertaining and giving the facts of the matter.

\subsection{Secondary Data Collection}

Official website of Dubai Airports and Abu Dhabi National Energy Company and other related websites to collect information about various related topics mentioned in the study.

\section{Data Analysis (Results) \& Discussions}

\subsection{Interviews}

1) Hypothesis (H1) Questions and Responses obtained from the SCADA \& Power Generation Department for the purpose of knowing to set the frequency in Maximo.

Q1. How do you schedule your assets for maintenance? 
According to time intervals, as, for examples every 500 running hours or every 3 months depending on the asset requirements.

Q2. Did the implementation of Maximo affect the way of operation?

It is organized in a seamless manner and provides assets maintenance history and when the next maintenance is due.

Q3. As assets age, do they require changing the frequency of the maintenance?

It is solely dependent on the asset as it may encounter faults over time and require close attention, Riaz added that even identical assets with the same manufacturing date can perform differently and one might treat them in different ways, according to the requirement, as for example, reduce the maintenance frequency for that particular asset only. As it is a life-case, Maximo is not able to understand the reasons for changing the maintenance dates.

Q4. How do you treat the above phenomena currently and is it satisfactory?

A New Work Order (WO), is created to open up an investigation on that asset and maintain it away from the original preventive maintenance schedule. It acts like a hidden maintenance done to this asset and it is not shown as part of the maintenance plan since it does not fall within the set frequency.

No, it is not, as it does not project actual data on that asset, On the contrary it does not give any indication of any fault, showing the asset is working according to the norms.

Q5. What if Maximo is able to capture vibration analysis \& thermal imagery data for a particular asset and set flexible maintenance dates accordingly?

That is a dream come true, and hope Maximo will integrate to reach to that level one day, as our assets are in need for a flexible maintenance regime as they age and encounter problems which forces us to deviate from the set targets. Through introducing capturing methods such as vibrational analysis and thermal imagery into Maximo trends can be plotted and cases can be shown, as to which asset is operating under the norms and which requires close attention. Therefore, set the dates according to the asset needs rather than a fixed frequency.

2) Hypothesis (H2) and Responses obtained from the different people from different fields, from procurement, Finance \& Business support for the purpose of how Maximo can integrate common spare parts for different departments under one grouping.

Q1. Maximo shows several identical spare parts in the dashboard which creates confusion to end users and why can't they be clubbed under a single line item?

One respondent stated that every department has identified its requirement of spare parts and it just happens that many departments use the same part's therefore, every spare part is registered under a different department with a minimum and maximum requirement which clearly differs from one department to another. On the other hand, other interviewees answered that, they release the approval for procuring the spare parts for every department separately, 
Also, from procurement side, the interviewee has claimed that they don't have an issue at all, as long as we have the confirmation we do procure the common spare part from the same supplier.

Q2. Assuming after years of operations and cycles, going through the history of common spare parts ordered and used can Maximo predict or somehow project the future use?

From Business support side, it was found that most certainly it could do that, a figure can be obtained. However, it calls for re-engineering of Maximo for dynamic times.

Q3. Related to the above question, what good will it do to you?

From procurement side, it was found that knowing the full year capacity and requirement will improve two main aspects in our efficiency. First is reducing the number of processes per year, since each department raises a request with quantity requirement for a certain time and may request again after a couple of months whereas if raised once and for all departments it would reduce significantly to one process per year and that will reduce the number of reports Maximo generates, saving memory space too. Second, it will lead to a dedicated inventory space for common spare parts with a reserved quantity for each department rather than having the same spare part stored in different locations just because it has a different unique code, which occupies space.

Q4. Related to the above question, is that option feasible although it is a common spare part yet it belongs to different department's budget?

From Finance side, the number of transactions and data points through Maximo will decrease, improving both store inventory and memory space. Yet, the complication exists when it comes to each department's expenditure. Honestly, there is a way of having a separate cost center for common spare parts to be procured which deducts automatically through Maximo from each departments budget according to usage, it would turn out to be helpful. But, it is a matter to be decides at the management level.

\subsection{Results and Discussions}

Based on the literature review and interviews conducted, the Hypothesis (H1), results are quite appealing from both ends. End users agree on the usability of sensors and gadgets to improve the efficiency, since the current system is not supporting such additives which increase the efficiency and reliability of the assets within the organizations. Yet, a future proposal is applicable upon further investment and integration of sensors into Maximo. The System Engineers (end users) from both companies Mr. Riaz \& Mr. Nizam second the means of modern monitor systems to best capture the best practice and current system is overridden due to the absence of such additives. Mr. Nizam added that monitoring data trends can be used in a manual manner currently but would be a hassle of records per department's keep. The literature review illustrates the movement towards an intelligent flexible maintenance schedules to obtain a higher rate of 
efficiency and reliability, but the investment to create the link of the monitor trends is to be considered as the next step in increasing the efficiency of the Maximo.

The Hypothesis (H2), obtained from the secondary data showed grouping of common spare parts is hectic and raises an issue of complications to end users, although some utilize dedicated software, from the interviews with business support, procurement, and finance departments representatives, the problem is mainly identified as mainly financial when relating to software, as the separate budgets are allocated and can't be crossed.

Proposing a solution relating to the software implication along with a managerial intervention, to come up with a solution to best attain departments' needs and solves financial problems such as easing procurement measures and reducing data points and processes related to Maximo/business support. A separate account is to be created and integrated to Maximo, retrieving historical trends of spare parts consumption over the years and forecasting future requirements and procuring them with a single shot process; moreover, departments acquiring those spare parts through Maximo Material Requisition portal will automatically deduct from the shared account in respect of the department's budget. This step will not only reduce process points intact with finance but also reduce procurement and supplier processes and therefore inventory keeping, which will enhance the efficiency of materials flow to departments.

\section{Conclusions}

The Engineering Service Unit at Dubai Airports, and the Abu Dhabi National Energy Company, including seven power and water plants in the country, as well as many other companies within the UAE have adopted Maximo, an IBM integrated Software used to manage their resources in a manner that is in harmony with their business strategy. The Maximo system is a type of I.S which helps organizations to provide a link between cross-functional departments and teams. Many of the organizations in the UAE using Maximo have faced several difficulties during implementation because the software needs to be customized to fit the engineering requirements, therefore improvements to the system are valid and have been addressed with a proposed solution. Based on literature review and interviews, this paper examined the efficiency of Maximo system in Dubai Airports and Abu Dhabi National Energy Company, looking at the possibilities of improvement and coping with modern techniques adapted worldwide.

Creating a strategy on how to improve the efficiency of the Maximo is critical for the Dubai Airports, and Abu Dhabi National Energy Company, creating a comprehensive grouping of different items from various departments with minimum quantities, financial standing, budget, procurement teams, and the buffer store locations have been reviewed by several studies as a means of improving the Maximo information system. The aim of adapting a flexible system is importing alarming gadgets into the system and integrating them into Max- 
imo, to free the software from the fixed scheduled maintenance, to flexible systems reduce manufacturing expenses and increases asset reliability and efficiency. Additionally, Intelligent Maintenance Systems are to be introduced to collect data to predict future failures; through analyzing the behavior of the machines through gadgets/sensors, that shift from fixed maintenance regimes to flexible "Smart" maintenance schedules. This was not the case for Dubai Airports and Abu Dhabi National Energy Company with Maximo.

To get a better grasp of the challenges of Maximo at the Dubai Airports and Abu Dhabi National Energy Company, this study used interviews and literature review of similar problems in other organizations. End users agreed on the usability of sensors and gadgets to improve the efficiency, since the current system is not supporting such additives that increase the efficiency and reliability of the assets within the organizations yet, a future proposal is applicable upon further investment and integration of sensor readings to Maximo. Regarding the second Hypothesis, secondary data obtained showed grouping of common spare parts was hectic and raised the issue of complications to end users although some utilized dedicated software. Through the interviews with business support, procurement, and finance departments' representatives the problem was mainly identified as financial when relating to software as separate budgets are allocated and cannot be crossed. The proposed solution is to create a separate account and integrate it into Maximo: This account will help to retrieve historical trends of spare parts consumption and forecast future requirements through the intervention of managerial role into adaptation of process change from multiple process, into a single process, reducing the number of data points and improving material grouping and inventory keeping.

In the end, it is a modest but a significant contribution to optimum resource management in the iconic UAE companies of Dubai Airports and Abu Dhabi National Energy Company, which is applicable to all firms in general in the United Arab Emirates, a model of free-enterprise development in the resurgent Arab world. A good resource management at the Centre of the health of any enterprise is whether with the Maximo or non-Maximo. And of all, the UAE is admirably intent on it for nature, and resource conservation, well explaining its competitiveness and operational efficiencies, due to an admirable state leadership.

\section{Acknowledgements}

The Authors would like to thank 1) Mashaal Al Marzooqi, Associate of Asset Management at Abu Dhabi National Energy Company "TAQA"; 2) Ahmed Al Rahma, Operation and Maintenance Engineer at Dubai Airports 3) Muna Al Ali, Operation and Maintenance Engineer at Dubai Airports for their support in gathering the data and information.

\section{References}

[1] Amponsah, C. and Ahmed, G. (2017) New Global Dimensions of Business Excel- 
lence. International Journal of Business Excellence, 13, 60-78.

[2] Ahmed, G. (2017) Destination 2021. Forbes Middle East Guide, 46-47.

[3] Ahmed, G. (2018) Transformation of UAE from Desert to Developed Economy. Forbes Middle East, 70, 29.

[4] Baek, J.G. (2007) An Intelligent Condition-Based Maintenance Scheduling Model. The International Journal of Quality \& Reliability Management, 24, 312-327. https://doi.org/10.1108/02656710710730898

[5] Heart-Rate Monitors (2003) Consumers Union of US, Inc., Yonkers.

[6] Winter, D., Zoia, D.E., Zachary, K. and Wielgat, A. (2001) Rethinking Platform Engineering. Ward's Auto World, 37, 46-50.

[7] JAMA and Archives Journals (2009) Study of Hospital Relocation Provides Insights to Aid in Disaster Planning.Biotech Week, 3280.

[8] Jen, H., Shew, S., Atkinson, J., Rosenthal, J. and Hiatt, J. (2009) Creation of Inpatient Capacity during a Major Hospital Relocation Lessons for Disaster Planning. Jama Surgery, 144, 859-864. https://doi.org/10.1001/archsurg.2009.146

[9] Kulus, J. (2012) Should the MDS Department Be Centralized? Long-Term Living, $61,20$.

[10] Doyle, K., Saulman, J. and Cary, B. (2014) IBM Solution Approach for IBM Enterprise Asset Management. International Business Machines Corporation. Maximo EAM Use Planning Pattern White Paper, IBM Corporation Software Group, Somers, 1-29.

[11] Pyles, R., Tripp, R., Lynch, K., Snyder, D., Mills, P. and Drew, J. (2008) A Common Operating Picture for Air Force Materiel Sustainment: First Steps, Santa Monica, CA, RAND Corporation. https://doi.org/10.7249/MG667

[12] Beavers, D. (1997) Spare Part Surgery. Supply Management, 2, 34-35.

[13] de Souza, R., Albert Wee, K.T., Othman, H. and Garg, M. (2011) A Proposed Framework for Managing Service Parts in Automotive and Aerospace Industries. Benchmarking, 18, 769-782. https://doi.org/10.1108/14635771111180699

[14] Hyvönen, H. (2014) Operations \& Maintenance Business Model Transformation-Multiple Case Studies. Modern Economy, 5, 1161-1170.

https://doi.org/10.4236/me.2014.513108

[15] Harrington, Y. (2015) Deep Dive into Predictive Inventory Optimization, IBM Maximo Asset Management, IBM Developer Works.

https://www.ibm.com/developerworks/community/wikis/home?lang=en\#!/wiki/IB M\%20Maximo\%20Asset\%20Management/page/Deep\%20dive\%20into\%20predictiv e\%20inventory\%20optimization

[16] Batty, B., Borges, D., Costa, B., Faustino, J., Ferreira, A., Filho, H., Filho, D., Gucer, V., Kipel, M., Labadi, Z., Murasaki, E., Pedrozo, J., Silva, V., Silveira, M. and Tamarindo, P. (2010) Implementing IBM Maximo for Service Providers. IBM Corporation, International Technical Support Organization, Poughkeepsie, 1-8.

[17] Tags, R., Sage, P., Osana, M., Tepora, D. and de Asis, J. (2011) The Maximo Manager's Guide to Business Performance Management. Reliability web.com Press, Fort Myers. 


\section{Appendix}

\section{Interviews questions:}

\section{Hypothesis I.}

Engineer Riaz Mubarak from SCADA Department \& Engineer Siddique Nizzam from Power Generation Department were interviewed about setting the frequency in Maximo.

Q1. How do you schedule your assets for maintenance?

Q2. Did the implementation of Maximo affect the way of operation?

Q3. As assets age does it require to change the frequency of the maintenance?

Q4. How do you treat the above phenomena currently and is it satisfactory?

Q5. What if Maximo is able to capture vibration analysis \& thermal imagery data for a particular asset and set flexible maintenance dates accordingly?

\section{Hypothesis II.}

Mr. Adil from procurement, Mr. Hiren from Finance, Mr. Srinivas from Business support were interviewed on how Maximo can integrate common spare parts for different departments under one grouping.

Q7. Maximo shows several identical spare parts in the dashboard, it creates a confusion to end users why can't they be clubbed under a single line item?

Q8. Assuming after years of operations and cycles, through going after the history of common spare parts ordered and used can Maximo predict or somehow project the future use?

Q9. Related to the above question, what good will it do you?

Q10. Related to the above question, is that option feasible although it is a common spare part yet it belongs to a different department's budget? 